\title{
Phase II Study of Preoperative Concurrent Chemoradiotherapy with S-1 plus Bevacizumab for Locally Advanced Resectable Rectal Adenocarcinoma
}

\author{
Sotaro Sadahiro ${ }^{a}$ Toshiyuki Suzuki ${ }^{a}$ Akira Tanaka ${ }^{a}$ Kazutake Okada ${ }^{a}$ \\ Gota Saito $^{a}$ Akemi Kamijo $^{a}$ Takeshi Akibab Shuichi Kawadab $^{b}$
}

Departments of a Surgery and ${ }^{b}$ Radiology, Tokai University, Isehara, Japan

\section{Key Words}

Rectal cancer · Chemoradiotherapy · Phase II study · S-1 · Bevacizumab

\begin{abstract}
Objective: A single-arm phase II clinical trial was conducted to evaluate the safety and efficacy of preoperative chemoradiotherapy (CRT) with concurrent S-1, bevacizumab, and radiation in patients with locally advanced rectal cancer (LARC). Methods: Fifty-two patients with LARC were enrolled. A total dose of 45 Gy was delivered in 25 fractions over 5 weeks, S- 1 was administered orally twice a day on days 1-14 and 22-35, and bevacizumab was administered on days 1,15 , and 29 . Surgical resection was scheduled 8 weeks (6-10 weeks) after completing the CRT. Results: All 52 patients underwent RO radical surgery. Sphincter preservation was possible in 38 (73.1\%) patients. A pathologic complete response was obtained in 10 (19.2\%) patients, a pathologic downstaging was achieved in 37 (71.2\%) patients, and the tumor shrinkage rate was $77.1 \%$. The only grade 3 adverse events were leukopenia and rash in 1 (1.9\%) patient. The rate of postoperative complications was $28.8 \%$. Anastomotic leakage occurred in $9(23.7 \%)$ of the 38 patients who underwent sphincter-preserving surgery. Perineal wound dehiscence developed in 2 (14.3\%) of the 14 patients who received an abdominoperineal resection. Conclusions: Adding bevacizumab to S-1 clearly increased the incidence of woundrelated complications, with no distinct enhancement of tumor response.
\end{abstract}




\section{Oncology}

\begin{tabular}{l|l}
\hline Oncology 2015;88:49-56 \\
\hline DOI: 10.1159/000367972 & $\begin{array}{l}\text { @ 2014 S. Karger AG, Basel } \\
\text { www.karger.com/ocl }\end{array}$ \\
\hline
\end{tabular}

Sadahiro et al.: Phase II Study of Preoperative Concurrent Chemoradiotherapy with S-1 plus Bevacizumab for Locally Advanced Resectable Rectal Adenocarcinoma

\section{Introduction}

For patients with clinical stage II and III rectal cancer, preoperative treatment with radiotherapy and 5-fluorouracil (5-FU)-based chemotherapy is recommended [1]. Capecitabine, an oral fluoropyrimidine, can be regarded as a convenient alternative to 5-FU in patients who receive preoperative chemoradiotherapy (CRT) for locally advanced rectal cancer (LARC) [2].

$\mathrm{S}-1$ is an oral anticancer drug that combines tegafur, a prodrug of 5-FU, with 5-chloro2,4-dihydropyrimidine (CDHP) and potassium oxonate (Oxo) in a molar ratio of 1:0.4:1 [3, 4]. We previously performed a phase I/II study of preoperative concurrent CRT with S-1 in patients with LARC and reported that the effectiveness of CRT with S-1 was similar to that of CRT with capecitabine. Moreover, CRT with S-1 was associated with mild adverse events, with no hand-foot syndrome [5].

Bevacizumab is a monoclonal antibody to vascular endothelial growth factor (VEGF) that is used to treat advanced colorectal cancer. Preclinical models have suggested that the induction of VEGF by radiation contributes to tumor radioresistance and that anti-VEGF monoclonal antibody treatment can compensate for resistance to radiation [6, 7]. Preoperative CRT combining bevacizumab with 5 -FU or capecitabine is thus considered promising for the management of LARC, but adequate evidence supporting the efficacy and safety of such regimens is lacking [8-10].

The aim of this single-arm phase II clinical trial was to evaluate the safety and efficacy of preoperative CRT with concurrent S-1, bevacizumab, and radiation in patients with LARC. This study was approved by the Institutional Review Board of our university (10R-113), and all patients provided their written informed consent.

\section{Patients and Methods}

\section{Study Design}

This study was a nonrandomized, single-institution, phase II trial designed to evaluate the feasibility, safety, and efficacy of neoadjuvant radiotherapy with concurrent oral S-1 and bevacizumab in patients with locally advanced resectable rectal adenocarcinoma. The primary endpoint was the pathologic complete response (pCR) rate. We also evaluated acute toxicity, perioperative morbidity, the degree of tumor shrinkage, and the downstaging rate as secondary endpoints.

\section{Eligibility Criteria}

Patients with a histologically confirmed diagnosis of adenocarcinoma of the middle or lower rectum (cT3-T4, M0 or Tx, N+, M0) were enrolled. Additional eligibility criteria were as follows: no prior chemotherapy or pelvic radiation; an Eastern Cooperative Oncology Group performance status of $\leq 1$; age 20-80 years; adequate organ function, as defined by a leukocyte count of $\geq 4,000$ to $\leq 12,000 / \mathrm{mm}^{3}$, a neutrophil count of $\geq 2,000 / \mathrm{mm}^{3}$, a platelet count of $\geq 100,000 / \mathrm{mm}^{3}$, a hemoglobin level of $\geq 9 \mathrm{~g} / \mathrm{dl}$, a serum bilirubin level of $\leq 1.5 \mathrm{mg} / \mathrm{dl}$, serum aspartate aminotransferase and alanine aminotransferase levels of $\leq 100 \mathrm{U} / \mathrm{l}$, a serum creatinine level of $\leq 1.1 \mathrm{mg} / \mathrm{dl}$ (for men) or $\leq 0.7 \mathrm{mg} / \mathrm{dl}$ (for women), and a creatinine clearance of $\geq 50$ $\mathrm{ml} / \mathrm{min}$; the ability to ingest food and drugs orally; and no high medical risks.

\section{Radiotherapy}

A total irradiation dose of 45 Gy was delivered in daily fractions of 1.8 Gy, 5 days per week for 5 weeks, using a four-field box technique. The patients were irradiated using a 15-MV linear accelerator. All patients underwent computed tomography (CT) simulation for three-dimensional conformal radiotherapy. The gross tumor volumes of the primary rectal mural tumor and lymph nodes $>1.0 \mathrm{~cm}$ in diameter were visualized on CT and magnetic resonance imaging (MRI). The clinical target volume included the gross tumor volume, mesorectum, presacral space, and the entire sacral hollow and regional lymphatics, including the perirectal, internal iliac, and presacral lymphatics. The planning target volume was located $1 \mathrm{~cm}$ outside of the clinical target volume. In accordance with these definitions, the treatment fields were set as follows: the lateral 


\section{Oncology}

\begin{tabular}{l|l}
\hline Oncology 2015;88:49-56 & $\begin{array}{l}\text { @ 2014 S. Karger AG, Basel } \\
\text { www.karger.com/ocl }\end{array}$ \\
\hline DOI: $10.1159 / 000367972$ &
\end{tabular}

Sadahiro et al.: Phase II Study of Preoperative Concurrent Chemoradiotherapy with S-1 plus Bevacizumab for Locally Advanced Resectable Rectal Adenocarcinoma

borders of the planning target volume were $1.5 \mathrm{~cm}$ lateral to the widest bony margin of the true pelvic wall; the superior border was placed at L5-S1; the inferior border within the anal canal; the anterior border at the most posterior aspect of the symphysis pubis, and the posterior border at the most posterior aspect of the sacrum. All patients underwent three-dimensional treatment planning.

\section{Chemotherapy}

S-1 was given orally twice a day (after breakfast and dinner) on days 1-14 and 22-35. The initial dose of S-1 was the standard dose used for monotherapy $\left(80 \mathrm{mg} / \mathrm{m}^{2} /\right.$ day). Bevacizumab was administered at $5 \mathrm{mg} / \mathrm{kg}$ body weight on days 1, 15, and 29.

\section{Safety Assessment and Dose Modifications}

The pretreatment evaluation included a clinical history and physical examination, complete blood cell count, blood chemistry, chest and abdominopelvic CT, colonoscopy with biopsy, contrast barium enema examination, pelvic MRI, and transrectal ultrasonography. Toxic effects were assessed every week during therapy and for 2 weeks after the end of treatment. A physical examination, blood cell count, and blood chemistry were conducted, and the results were recorded. The following recommendations were used for dose modification: if a patient had hematologic toxicity (grade 3 or higher leukopenia or neutropenia or grade 2 or higher thrombocytopenia) or nonhematologic toxicity (grade 3 or higher), S-1 chemotherapy was temporarily discontinued until the toxicity resolved to grade 2 or lower, and treatment was restarted at a lower dose of S-1 (65 mg/m²/day); bevacizumab was withdrawn in the event of thrombosis, perforation of the gastrointestinal tract, or bleeding (grade 3 or higher).

\section{Surgery and Postoperative Adjuvant Chemotherapy}

Surgical resection was scheduled to be performed 8 weeks (6-10 weeks) after completing the CRT. The surgical techniques included low anterior resection and abdominoperineal resection, performed using mesorectal resection techniques. Postoperative adjuvant chemotherapy, consisting of 8 courses of S-1 for 2 weeks repeated every 3 weeks, was recommended but was not included in the study protocol.

\section{Evaluation of Tumor Response and Tumor Shrinkage}

Tumor response was evaluated on the basis of the histologic findings of resected specimens. The tumor regression grade (TRG) was used to evaluate histologic regression [11]. A pathologic tumor response was determined by the presence of pathologic downstaging or a pCR.

Double-contrast barium enema examination and MRI volumetry were performed before CRT and immediately before surgery. A 1.5-tesla MRI system with a surface coil was used. Before the MRI, colonic irrigation was performed, and then barium was infused. Cross-sectional areas were measured on axial T2-weighted images. The degree of tumor shrinkage on barium enema examination was calculated by measuring the tumor along the major axis (length along the long axis of the bowel) on lateral views. The methods used to measure the degree of tumor shrinkage have been described previously [12-14].

\section{Early Stopping Rule and Statistical Analysis}

Since we had no experience using radiotherapy combined with S-1 and bevacizumab, the study was scheduled to be prematurely terminated if the incidence of grade 4 or higher treatment-related adverse events was $>8.19 \%$. The incidence of $8.19 \%$ was based on the frequency of grade 4 or higher serious adverse reactions in specific use surveillance performed after bevacizumab was approved for the treatment of curatively unresectable advanced or recurrent colon cancer and rectal cancer in Japan (patients enrolled: 2,712; patients included in the analysis: 2,698) [15]. In that study, the numbers of enrolled patients in whom treatment was prematurely terminated and the numbers of events were as follows: 10 patients, 3 events; 20 patients, 5 events; 30 patients, 7 events; 40 patients, 8 events, and 50 patients, 9 events.

Preoperative CRT with regimens combining bevacizumab with 5-FU or capecitabine has been reported to have a pCR rate of 9-32\% in patients with LARC [8-10]. We previously obtained a pCR rate of $22 \%(95 \%$ confidence interval 8.6-42.3) in patients who received CRT with S-1 alone [5]. In the present study, we assumed that the pCR rate would be $25 \%$, with a minimum activity level pCR rate of $10 \%$, an $\alpha$ level of 0.05 (two-sided), and a $\beta$ level of 0.20 . We estimated that 49 patients would be required. The target number of patients was therefore set at 52, taking excluded patients and dropouts into account. 


\section{Oncology}

\begin{tabular}{|c|c|}
\hline \multicolumn{2}{|l|}{ Oncology 2015;88:49-56 } \\
\hline DOI: $10.1159 / 000367972$ & $\begin{array}{l}\text { (c) } 2014 \text { S. Karger AG, Basel } \\
\text { www.karger.com/ocl }\end{array}$ \\
\hline \multicolumn{2}{|c|}{$\begin{array}{l}\text { Sadahiro et al.: Phase II Study of Preoperative Concurrent Chemoradiotherapy with S- } \\
\text { plus Bevacizumab for Locally Advanced Resectable Rectal Adenocarcinoma }\end{array}$} \\
\hline $\begin{array}{l}\text { Median age (range), years } \\
\text { Gender }\end{array}$ & $59(32-73)$ \\
\hline Male/female & $39(75) / 13(25)$ \\
\hline Performance status & \\
\hline $0 / 1$ & $43(83) / 9(17)$ \\
\hline $\begin{array}{l}\text { Median distance from the } \\
\text { anal verge (mean } \pm \text { SD), } \mathrm{cm}\end{array}$ & $5.5(5.1 \pm 2.3)$ \\
\hline Site & \\
\hline $\begin{array}{l}\text { Middle/lower rectum } \\
\text { Histologic type differentiation }\end{array}$ & $26(50) / 26(50)$ \\
\hline Well/moderate/poor & $33(63) / 18(35) / 1(2)$ \\
\hline cStage & \\
\hline${ }_{\mathrm{cT}}{ }^{\mathrm{II} / \mathrm{III}}$ & $16(31) / 36(69)$ \\
\hline $2 / 3 / 4$ & $2(4) / 49(94) / 1(2)$ \\
\hline $\mathrm{cN}$ & \\
\hline $0 / 1$ & $16(31) / 36(69)$ \\
\hline
\end{tabular}

Table 1. Patient demographics $(\mathrm{n}=52)$

\section{Results}

\section{Patients}

A total of 52 patients were enrolled from February 2011 through September 2013. The patients' characteristics are shown in table 1 . Sixteen patients (31\%) had clinical stage II disease, and 36 (69\%) had clinical stage III disease.

\section{Toxicity and Compliance with Treatment}

The main adverse events are shown in table 2. Dose reduction and transient withdrawal of irradiation were performed because of adverse events in only 1 patient. In this patient, the dose of S-1 was reduced because grade 3 leukopenia occurred on day 7 of treatment, and the lower dose of S-1 was given until day 14. S-1 was not given on days 15-21, as originally scheduled. Treatment with the reduced dose of S-1 was resumed on day 22, but a grade 3 rash developed on day 28, and both S-1 and radiotherapy were discontinued. Only radiotherapy was resumed on day 35 and was completed (the patient received a total dose of 45 Gy in 25 fractions). Bevacizumab was administered 3 times, as scheduled. The other patients had no grade 3 or higher adverse events. Grade 2 anal pain developed in 24 (46.2\%) patients.

\section{Surgery}

All 52 patients underwent radical surgery. Sphincter-preserving surgery was performed in $38(73.1 \%)$ of the 52 patients. All patients received $\mathrm{R} 0$ resection. The median interval between completing the radiotherapy and surgery was 55 days (range 38-70).

\section{Tumor Response and Postoperative Complications}

Among the 52 patients, 10 had a pCR. The pCR rate was thus $19.2 \%$ (95\% confidence interval 9.6-32.5). One patient had complete regression of the primary tumor, but a residual tumor was found in the perirectal lymph nodes. Histologic regression was evaluated to be TRG 1 in 11 (21.2\%) patients and TRG 2 in 12 (23.1\%) patients; marked tumor regression (TRG 1 or 2) was thus obtained in $23(44.2 \%)$ of the 52 patients (table 3). In addition, 27 (51.9\%) patients achieved $\mathrm{T}$ downstaging, and 25 (48.1\%) patients achieved $\mathrm{N}$ downstaging, accounting for a combined pathologic downstaging rate of $71.2 \%$ (37 of the 52 patients). The 


\section{Oncology}

Table 2. Adverse events in the 52 patients

Table 3. Histologic tumor regression in the 52 patients

Table 4. Degree of tumor shrinkage in the 52 patients

\begin{tabular}{l|l}
\hline Oncology 2015;88:49-56 & $\begin{array}{l}\text { C } 2014 \text { S. Karger AG, Basel } \\
\text { www.karger.com/ocl }\end{array}$ \\
\hline DOI: $10.1159 / 000367972$ & $\begin{array}{l}\text { Sadahiro et al.: Phase II Study of Preoperative Concurrent Chemoradiotherapy with S-1 } \\
\text { plus Bevacizumab for Locally Advanced Resectable Rectal Adenocarcinoma }\end{array}$
\end{tabular}

\begin{tabular}{lcrrrll}
\hline & All & \multicolumn{2}{l}{ Grade } & & \\
\cline { 3 - 7 } & & 1 & 2 & 3 & 4 & $3 / 4$ \\
\hline Hematologic adverse events & & & & & \\
Leukopenia & $28(53.8)$ & 13 & 14 & 1 & 0 & $1(1.9)$ \\
Neutropenia & $17(32.7)$ & 13 & 4 & 0 & 0 & 0 \\
Anemia & $16(30.8)$ & 9 & 7 & 0 & 0 & 0 \\
Thrombocytopenia & $1(1.9)$ & 0 & 1 & 0 & 0 & 0 \\
Nonhematologic adverse events & & & & & \\
AST/ALT & 0 & 0 & 0 & 0 & 0 & 0 \\
Anorexia & $23(44.2)$ & 13 & 10 & 0 & 0 & 0 \\
Vomiting & $1(1.9)$ & 1 & 0 & 0 & 0 & 0 \\
Diarrhea & $30(57.7)$ & 22 & 8 & 0 & 0 & 0 \\
Fatigue & $35(67.3)$ & 29 & 6 & - & - & - \\
Stomatitis & $4(7.7)$ & 3 & 1 & 0 & 0 & 0 \\
Rash & $1(1.9)$ & 0 & 0 & 1 & 0 & $1(1.9)$ \\
Hypertension & $3(5.8)$ & 0 & 3 & 0 & 0 & 0 \\
Anal pain & $31(59.6)$ & 7 & 24 & 0 & - & 0 \\
\hline
\end{tabular}

Values are given as numbers (\%).

\begin{tabular}{llc}
\hline TRG 1 & $\begin{array}{l}\text { (pCR, absence of viable cancer cells in the } \\
\text { resected specimen) }\end{array}$ & $11(21.2)$ \\
TRG 2 & (presence of residual cancer cells) & $12(23.1)$ \\
TRG 3 & (fibrosis outgrowing residual cancer cells) & $17(32.7)$ \\
TRG 4 & (residual cancer cells outgrowing fibrosis) & $11(21.2)$ \\
TRG 5 & (absence of response) & $1(1.9)$ \\
\hline
\end{tabular}

Values are given as numbers (\%).

\begin{tabular}{ll}
\hline Method of detection & Mean shrinkage \pm SD, $\%$ \\
\hline Barium enema (two-dimensional) & $51.8 \pm 16.4$ \\
MRI (three-dimensional) & $77.1 \pm 13.0$ \\
\hline
\end{tabular}

degree of tumor shrinkage was $51.8 \pm 16.4 \%$ as assessed two-dimensionally on barium enema examination and $77.1 \pm 13.0 \%$ as assessed three-dimensionally on MRI volumetry (table 4).

The postoperative complication rate was $28.8 \%$ (15 of the 52 patients). Anastomotic leakage occurred in $9(23.7 \%)$ of the 38 patients who underwent sphincter-preserving surgery. Perineal wound dehiscence developed in $2(14.3 \%)$ of the 14 patients who underwent abdominoperineal resection. A rectovaginal fistula was found in 1 (1.9\%) patient 2 months after surgery.

\section{Discussion}

Preoperative radiotherapy with concurrent 5-FU-based chemotherapy decreases the risk of local recurrence and remains a standard of care in patients with LARC. The achievement of a pCR and a negative circumferential resection margin correlate with a good prognosis and 


\section{Oncology}

\begin{tabular}{l|l}
\hline Oncology 2015;88:49-56 \\
\hline DOI: $10.1159 / 000367972$ & $\begin{array}{l}\text { @ 2014 S. Karger AG, Basel } \\
\text { www.karger.com/ocl }\end{array}$ \\
\hline
\end{tabular}

Sadahiro et al.: Phase II Study of Preoperative Concurrent Chemoradiotherapy with S-1 plus Bevacizumab for Locally Advanced Resectable Rectal Adenocarcinoma

are considered potential early markers of the efficacy of CRT [16]. In recent studies comparing the outcomes of preoperative CRT with 5-FU or capecitabine in patients with LARC, the pCR rate ranged from 11 to $18 \%$ for 5 -FU and from 16 to $30 \%$ for capecitabine, with no significant difference between the drugs [17-20]. In our previous study of preoperative CRT with S-1 alone, the pCR rate was $22 \%$, consistent with the results of previous studies [5].

Antiangiogenic therapy has been an important strategy in cancer treatment since the time that bevacizumab combined with 5-FU-based chemotherapy was shown to significantly improve overall survival in patients with metastatic colorectal cancer [21]. Willett et al. [22] were the first to show that bevacizumab monotherapy has clinically significant antivascular and vascular-normalizing effects in rectal cancer. They obtained a pCR rate of 16\% (5 of 32 patients) in a phase II study of bevacizumab plus preoperative 5-FU and radiation in LARC [9]. Subsequently, Spigel et al. [23] obtained a pCR rate of 29\% (10 of 35 patients) in a phase II study of preoperative 5-FU-based CRT plus bevacizumab. Recently, an increasing number of studies have evaluated preoperative CRT with capecitabine plus bevacizumab in patients with LARC. Marijnen et al. [8] obtained a pCR rate of $9 \%$ (2 of 23 patients) in an interim analysis of a multicenter clinical trial. In subsequent phase II studies, pCR rates of $32 \%$ ( 8 of 25 patients) were reported by Crane et al. [10], 13\% (8 of 61 patients) by Velenik et al. [24], and $14 \%$ ( 6 of 43 patients) by Gasparini et al. [25]. In our study, the pCR rate was $19.2 \%$ (10 of 52 patients), which was generally consistent with these values.

In previous studies of preoperative fluoropyrimidine-based CRT, we obtained tumor shrinkage rates of 69\% [5] and 71\% [14] on MRI. In the present study, the tumor shrinkage rate was $77 \%$, which was in accord with previous results.

As mentioned above, the tumor response in patients with LARC who received preoperative CRT with S-1 plus bevacizumab was comparable to the response previously obtained by combining bevacizumab with 5-FU or capecitabine. However, a clinically significant potentiation of the tumor response was not apparent in those patients who received bevacizumab in addition to S-1, contrary to our expectations.

In our study, adverse events were extremely mild during CRT as well as during the period from completing the CRT to surgery. The only grade 3 or higher adverse events were leukopenia and rash (both grade 3 ) in $1(1.9 \%)$ patient during CRT. In our previous study, grade 3 diarrhea developed in $3(11.1 \%)$ of 27 patients who received CRT with S-1 [5]. In contrast, no patient who received CRT with S-1 plus bevacizumab had grade 3 or higher diarrhea in the present study. However, grade 1 or 2 anal pain was reported by $31(59.6 \%)$ of the 52 patients, and $24(46.2 \%)$ patients had grade 2 anal pain. Anal pain did not occur in patients with LARC who received CRT with S-1 alone [5]. The incidence of proctitis/proctalgia was reported to be $18.8 \%$ ( 6 of 32 patients) in patients who received CRT with 5 -FU plus bevacizumab in a study by Willett et al. [9] and 20.9\% (9 of 43 patients) in patients who received CRT with capecitabine plus bevacizumab in a study by Gasparini et al. [25]. However, since many studies did not report the occurrence of proctitis or proctalgia, these complications were apparently not problematic in previous studies of CRT. The mechanism underlying the increase in anal pain associated with the concomitant use of bevacizumab remains unknown.

In the present study, anastomotic leakage developed in $9(23.7 \%)$ of the 38 patients who underwent sphincter-preserving surgery, and perineal wound dehiscence developed in 2 $(14.3 \%)$ of the 14 patients who underwent abdominoperineal resection, indicating a trend toward a high rate of wound-related complications after surgery. In our previous study of patients who received CRT with S-1 alone, the rates of suture failure and delayed wound healing were $4.3 \%$ ( 1 of 23 patients) and $13.3 \%$ ( 4 of 30 patients), respectively [5]. In another study on CRT with capecitabine plus bevacizumab, Crane et al. [10] reported that $2(20.0 \%)$ of 10 patients had suture failure after low anterior resection, 2 (33.3\%) of 6 patients had delayed wound healing, and $2(33.3 \%)$ of 6 patients had perineal wound dehiscence after 


\section{Oncology}

\begin{tabular}{l|l}
\hline Oncology 2015;88:49-56 & \\
\hline DOI: $10.1159 / 000367972$ & $\begin{array}{l}\text { ○ 2014 S. Karger AG, Basel } \\
\text { www.karger.com/ocl }\end{array}$ \\
\hline
\end{tabular}

Sadahiro et al.: Phase II Study of Preoperative Concurrent Chemoradiotherapy with S-1 plus Bevacizumab for Locally Advanced Resectable Rectal Adenocarcinoma

abdominoperineal resection. Velenik et al. [24] reported that among 60 patients who received CRT with capecitabine plus bevacizumab, including 42 patients who underwent sphincterpreserving surgery, the rate of delayed wound healing was $30.0 \%$ (18 patients), with a suture failure rate of $11.7 \%$ (7 patients). Although these studies are not phase III trials, these findings suggest that the concurrent use of bevacizumab might increase the risk of wound-related complications. Caution is therefore required.

Long-term effects of this combination regimen cannot be judged by this trial. Long-term follow-up results on survival and on local control are needed to determine the potential impact of adding bevacizumab to preoperative standard CRT. We also recognize that using pCR as surrogate marker in rectal cancer is problematic.

In conclusion, the major effect associated with adding bevacizumab to CRT for LARC was an increase in the incidence rate of wound-related complications. Although adverse events were extremely mild both during CRT and during the period from completing the CRT to surgery, the concurrent administration of bevacizumab with capecitabine- or S-1-based CRT apparently offers no clinically significant benefit in patients with LARC.

\section{Disclosure Statement}

The authors declare that they have no competing interests. None of the authors received any funding support for this work.

\section{References}

1 Willett CG, Czito BG, Bendell JC: Radiation therapy in stage II and III rectal cancer. Clin Cancer Res 2007; 13: 6903s-6908s.

-2 Hofheinz R-D, Wenz F, Post S, Matzdorff A, Laechelt S, Hartmann JT, Müller L, Link H, Moehler M, Kettner E, Fritz E, Hieber U, Lindemann HW, Grunewald M, Kremers S, Constantin C, Hipp M, Hartung G, Gencer D, Kienle P, Burkholder I, Hochhaus A: Chemoradiotherapy with capecitabine versus fluorouracil for locally advanced rectal cancer: a randomised, multicentre, non-inferiority, phase 3 trial. Lancet Oncol 2012;13:579-588.

-3 Shirasaka T, Shimamato Y, Ohshimo H, Yamaguchi M, Kato T, Yonekura K, Fukushima M: Development of a novel form of an oral 5-fluorouracil derivative (S-1) directed to the potentiation of the tumor selective cytotoxicity of 5-fluorouracil by two biochemical modulators. Anticancer Drugs 1996;7:548-557.

4 Schoffski P: The modulated oral fluoropyrimidine prodrug S-1, and its use in gastrointestinal cancer and other solid tumors. Anticancer Drugs 2004;15:85-106.

5 Sadahiro S, Suzuki T, Tanaka A, Okada K, Kamijo A, Murayama C, Akiba T, Nakayama Y: Phase I/II study of preoperative concurrent chemoradiotherapy with S-1 for locally advanced, resectable rectal adenocarcinoma. Oncology 2011;81:306-311.

-6 Gorski DH, Beckett MA, Jaskowiak NT, Calvin DP, Mauceri HJ, Salloum RM, Seetharam S, Koons A, Hari DM, Kufe DW, Weichselbaum RR: Blockade of the vascular endothelial growth factor stress response increases the antitumor effects of ionizing radiation. Cancer Res 1999;59:3374-3378.

7 Lee C-G, Heijn M, di Tomaso E, Griffon-Etienne G, Ancukiewicz M, Koike C, Park KR, Ferrara N, Jain RK, Suit HD, Boucher Y: Anti-vascular endothelial growth factor treatment augments tumor radiation response under normoxic or hypoxic conditions. Cancer Res 2000;60:5565-5570.

8 Marijnen CA, Rutten H, de Wilt H, Tesselaar ME, Nuyttens J, Remmelzwaal J, Punt CJ, Martijn H, Hospers GA, Cats A: Preoperative chemoradiotherapy regimen with capecitabine and bevacizumab in locally advanced rectal cancer: a feasibility study of the Dutch Colorectal Cancer Group (DCCG) (abstract). J Clin Oncol 2008; 26(suppl):15040.

$\checkmark 9$ Willett CG, Duda DG, di Tomaso E, Boucher Y, Ancukiewicz M, Sahani DV, Lahdenranta J, Chung DC, Fischman AJ, Lauwers GY, Shellito P, Czito BG, Wong TZ, Paulson E, Poleski M, Vujaskovic Z, Bentley R, Chen HX, Clark JW, Jain RK: Efficacy, safety, and biomarkers of neoadjuvant bevacizumab, radiation therapy, and fluorouracil in rectal cancer: a multidisciplinary phase II study. J Clin Oncol 2009;27:3020-3026.

10 Crane CH, Eng C, Feig BW, Das P, Skibber JM, Chang GJ, Wolff RA, Krishnan S, Hamilton S, Janjan NA, Maru DM, Ellis LM, Rodriguez-Bigas MA: Phase II trial of neoadjuvant bevacizumab, capecitabine, and radiotherapy for locally advanced rectal cancer. Int J Radiat Oncol Biol Phys 2010;76:824-830.

11 Mandard A-M, Dalibard F, Mandard J-C, Marnay J, Henry-Amar M, Petiot J-F, Roussel A, Jacob J-H, Segol P, Samama G, Ollivier J-M, Bonvalot S, Gignoux M: Pathologic assessment of tumor regression after preoperative chemoradiotherapy of esophageal carcinoma. Clinicopathologic correlations. Cancer 1994;73:2680-2686. 


\section{Oncology}

\begin{tabular}{l|l}
\hline Oncology 2015;88:49-56 \\
\hline DOI: 10.1159/000367972 & $\begin{array}{l}\text { (c) 2014 S. Karger AG, Basel } \\
\text { www.karger.com/ocl }\end{array}$ \\
\hline
\end{tabular}

Sadahiro et al.: Phase II Study of Preoperative Concurrent Chemoradiotherapy with S-1 plus Bevacizumab for Locally Advanced Resectable Rectal Adenocarcinoma

-12 Suzuki T, Sadahiro S, Fukasawa M, Ishikawa K, Kamijo A, Yasuda S, Makuuchi H, Ohizumi Y, Murayama C: Predictive factors of tumor shrinkage and histological regression in patients who received preoperative radiotherapy for rectal cancer. Jpn J Clin Oncol 2004;34:740-746.

$\rightarrow 13$ Suzuki T, Sadahiro S, Tanaka A, Okada K, Kamata H, Kamijo A, Murayama C, Akiba T, Kawada S: Biopsy specimens obtained 7 days after starting chemoradiotherapy (CRT) provide reliable predictors of response to CRT for rectal cancer. Int J Radiat Oncol Biol Phys 2013;85:1232-1238.

14 Suzuki T, Sadahiro S, Tanaka A, Okada K, Saito G, Kamijo A, Akiba T, Kawada S: Relationship between histologic response and the degree of tumor shrinkage after chemoradiotherapy in patients with locally advanced rectal cancer. J Surg Oncol 2014;109:659-664.

15 The final results: bevacizumab safety post-marketing large cohort survey (PMS) in 2712 Japanese colorectal cancer patients. Chugai Pharmaceutical Co., Ltd. http://chugai-pharm.jp/hc/ss/pr/safe/report/ava/index. html.

-16 Sanghera P, Wong DWY, McConkey CC, Geh JI, Hartley A: Chemoradiotherapy for rectal cancer: an updated analysis of factors affecting pathological response. Clin Oncol 2008;20:176-183.

-17 Yerushalmi R, Idelevich E, Dror Y, Stemmer SM, Figer A, Sulkes A, Brenner B, Loven D, Dreznik Z, Nudelman I, Shani A, FenigE: Preoperative chemoradiation in rectal cancer: retrospective comparison between capecitabine and continuous infusion of 5-fluorouracil. J Surg Oncol 2006;93:529-533.

18 Das P, Lin EH, Bhatia S, Skibber JM, Rodriguez-Bigas MA, Feig BW, Chang GJ, Hoff PM, Eng C, Wolff RA, Delclos ME, Krishnan S, Janjan NA, Crane CH: Preoperative chemoradiotherapy with capecitabine versus protracted infusion 5-fluorouracil for rectal cancer: a matched-pair analysis. Int J Radiat Oncol Biol Phys 2006;66:13781383.

19 Kim DY, Jung KH, Kim TH, Kim D-W, Chang HJ, Jeong JY, Kim YH, Son S-H, Yun T, Hong CW, Sohn DK, Lim S-B, Choi HS, Jeong S-Y, Park J-G: Comparison of 5-fluorouracil/leucovorin and capecitabine in preoperative chemoradiotherapy for locally advanced rectal cancer. Int J Radiat Oncol Biol Phys 2007;67:378-384.

20 Chan AK, Wong AO, Jenken DA: Preoperative capecitabine and pelvic radiation in locally advanced rectal cancer - is it equivalent to 5-FU infusion plus leucovorin and radiotherapy? Int J Radiat Oncol Biol Phys 2010; 76:1413-1419.

-21 Hurwitz H, Fehrenbacher L, Novotny W, Cartwright T, Hainsworth J, Heim W, Berlin J, Baron A, Griffing S, Holmgren E, Ferrara N, Fyfe G, Rogers B, Ross R, Kabbinavar F: Bevacizumab plus irinotecan, fluorouracil, and leucovorin for metastatic colorectal cancer. N Eng J Med 2004;350:2335-2342.

-22 Willett CG, Boucher Y, di Tomaso E, Duda DG, Munn LL, Tong RT, Chung DC, Sahani DV, Kalva SP, Kozin SV, Mino M, Cohen KS, Scadden DT, Hartford AC, Fischman AJ, Clark JW, Ryan DP, Zhu AX, Blaszkowsky LS, Chen HX, Shellito PC, Lauwers GY, Jain RK: Direct evidence that the VEGF-specific antibody bevacizumab has antivascular effects in human rectal cancer. Nat Med 2004;10:145-147.

-23 Spigel DR, Bendell JC, McCleod M, Shipley DL, Arrowsmith E, Barnes EK, Infante JR, Burris HA III, Greco FA, Hainsworth JD: Phase II study of bevacizumab and chemoradiation in the preoperative or adjuvant treatment of patients with stage II/III rectal cancer. Clin Colorectal Cancer 2012;11:45-52.

24 Velenik V, Ocvirk J, Music M, Bracko M, Anderluh F, Oblak I, Edhemovic I, Brecelj E, Kropivnik M, Omejc M: Neoadjuvant capecitabine, radiotherapy, and bevacizumab (CRAB) in locally advanced rectal cancer: results of an open-label phase II study. Radiat Oncol 2011;6:105-112.

25 Gasparini G, Torino F, Ueno T, Cascinu S, Troiani T, Ballestrero A, Berardi R, Shishido J, Yoshizawa A, Mori Y, Nagayama S, Morosini P, Toi M: A phase II study of neoadjuvant bevacizumab plus capecitabine and concomitant radiotherapy in patients with locally advanced rectal cancer. Angiogenesis 2012;15:141-150. 